\title{
Base Pressure Fluctuations on Typical Missile Configuration in Presence of Base Cavity
}

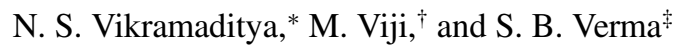 \\ National Aerospace Laboratories, Bangalore 560 017, India \\ and

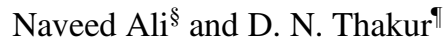 \\ Defense Research and Development Laboratory, Hyderabad 500 058, India
}

DOI: $\underline{10.2514 / 1 . A 33926}$

\begin{abstract}
Pressure fluctuations in the base region of a typical missile configuration at a freestream Mach number of 0.7 are examined experimentally in the presence and absence of a base cavity. The objective was to characterize the pressure fluctuations and explain the influence of base cavities on their behavior. Experiments include unsteady pressure measurements at six azimuthal locations. Substantial variation in the characteristics of pressure fluctuations is noticed along the azimuthal direction due to the asymmetry of the model. The base cavities are seen to enhance the base pressure and reduce the root mean square of the pressure fluctuations. Higher-order moments show diminishing trend as the length of the base cavity is increased. Spectra in the absence of cavity exhibit three types of narrow-band tones based on the Strouhal number. Type I tones disapper from the spectra for small-length cavities, whereas type II tones get suppressed. On the other hand, for the large-length cavities, type I tones get enhanced, whereas type II tones disappear. Autocorrelation and cross-correlation plots exhibit a large-amplitude, low-frequency oscillation that exists even after large separation times for the larger-length cavities, and virtually no such oscillations are visible for the small-length-cavity models.
\end{abstract}

\section{Nomenclature}

$C p_{b}=$ coefficient of base pressure, $\left(P_{b}-P_{\infty}\right) / Q_{\infty}$

$C p_{\text {rms }}=$ unsteady pressure coefficient, $p_{\text {rms }} / Q_{\infty}$

$D=$ diameter of base

$f \quad=$ frequency, $\mathrm{Hz}$

$G(S t)=$ power spectral density in terms of Strouhal number

$H=$ height of cavity

$M_{\infty}=$ freestream Mach number

$P_{\infty} \quad=$ freestream static pressure

$P_{b} \quad=$ mean static pressure on the base

$p \quad=$ instantaneous pressure

$p^{\prime} \quad=$ fluctuating pressure

$p_{\text {rms }}=$ root mean square of pressure fluctuation, $\sqrt{\overline{p^{\prime 2}}}$

$Q_{\infty} \quad=\quad$ dynamic pressure of freestream

$R_{x x}=$ autocorrelation coefficient,

$R_{x x}(x, 0, \tau)=\frac{\lim _{T \rightarrow \infty}(1 / 2 T) \int_{-T}^{T} p(x, t) p(x, t+\tau) \mathrm{d} \tau}{[p(x)]_{\text {ms }}^{2}}$

$R_{x y}=$ cross-correlation coefficient,

$R_{x y}(x, \xi, \tau)=\frac{\lim _{T \rightarrow \infty}(1 / 2 T) \int_{-T}^{T} p(x, t) p(x+\xi, t+\tau) \mathrm{d} \tau}{[p(x)]_{\mathrm{ms}}[p(x+\xi)]_{\mathrm{ms}}}$

St $\quad=$ Strouhal number, $f D / U_{\infty}$

$t=$ time, $\mathrm{s}$

$U_{\infty} \quad=$ freestream velocity

$\theta=$ circumferential or azimuthal angle

$\xi=$ separation distance between the measurement locations

$\tau \quad=\quad$ time lag, $\mathrm{s}$

\section{Introduction}

$\mathbf{T}$ HE topic of turbulent base flows continues to be an active area of research due to its significant impact on the performance and stability of an aerodynamic vehicle. Base flows are characterized by a massive separation occurring at the base due to an abrupt change in the rear geometry. The separated flow at the base region gives rise to two most undesirable issues: the unsteadiness at the base and an increase in the drag. The unsteadiness results in the unsteady loads and buffeting phenomena; the drag termed as "base drag" in some specific instances contributes to as high as $50 \%$ of the total drag. Several important factors influence the dynamics of the flow at the base; a few important among them are Mach number of the freestream, nature of the boundary layer ahead of the separation, and the afterbody geometry. Over the years investigations have been carried out to understand the base flows over a wide range of flow regimes, that is, subsonic to hypersonic speeds [1-ㅡ]. In spite of these efforts, the complete understanding continues to remain a distant reality. However, from the previous study [7] it is understood that the key elements that dictate the dynamics of the base flows are the development of shear layer and its reattachment, the recirculation region adjacent to the base, vortex shedding, and finally the interactions among the three. Numerous studies have been reported in the literature covering various aspects of the base flows. Nevertheless, these studies can be broadly classified into either those that focus on the structure and behaviour of the flow field in the vicinity of the base or those that investigate the techniques to control the flow, including those to mitigate the base drag.

The majority of these studies are aimed at understanding the important features and/or mechanisms of base flow and their behavior. A few works from the past decade have been discussed as follows. For example, Kawai and Fujii [1] from their numerical study identified the instability of the shear layer as a prominent mechanism from subsonic to supersonic Mach numbers. However, at supersonic Mach numbers they conclude that an additional mechanism of instability within the recirculation region comes into play. An experimental study carried out by Cannon et al. [8] suggests that the large pressure at the base corresponds to a large recirculation region and vice versa. Weiss et al. [9] from their numerical study on an axisymmetric turbulent separating and reattaching flow argue that large-scale coherent motions at a Strouhal number of 0.2 dominate an ordered structure. Their stability analysis indicates that the dynamics are dominated by a helical absolute instability. Schrijer et al. [10] from their investigations on an axisymmetric backward-facing step have shown that the flow field is 
dominated by the pulsing action of separated region leading to momentum exchange across the shear layer, flapping motion of the shear layer, and the presence of large circumfrential vortices.

Studies from the context of base flow control and base drag reduction show that several passive and active methods have been tested on both two- and three-dimensional bodies. These methods modify the flow field at the base to increase the base pressure and thereby reduce the base drag. For example, Choi et al. [11] and Zdravkovich [12] in their respective review papers present several passive and active flow control techniques for the bluff bodies. The most commonly used passive techniques are boat tailing, base cavities (solid and ventilated) [7,13,14], splitter plates, trapped vortex, and multistep and nonaxisymmetric afterbodies [7]. Active methods pursued include blowing $[\underline{15}, \underline{16}]$, base bleed [17]], Coanda effect [18], and so on. Among the passive devices listed, experience shows that the base cavities prove to be a promising method to reduce the base drag. However, the fluid dynamics associated with it is not well understood due to very limited investigations. Most of the early investigations into the base cavities focussed on their efficacy in controlling the base drag. Morel [19] has shown on an axisymmetric cylinder that both solid and ventilated base cavities have a potential to mitigate the base drag for a cavity depth below a certain value, beyond which the drag increases. He attributes the base drag reduction mechanism in the presence of cavity to the suppression of near-wake periodicity. Compton [20] from his experimental study on a stingmounted truncated cone has shown that the presence of cavity at the base increases the base pressure and thereby reduces the base drag. Viswanath [7] in the review paper suggested the following reasons for the base drag mitigation in the presence of a base cavity based on his and also from the experience of other researchers. For solid cavities, these include increased size of the recirculation region and thereby affecting the shear-layer entrainment, reduced length available for the shear-layer development and reduced influence at the base plane of the low pressure associated with the base vortex. On the other hand, for the ventilated cavities the increase in the base pressure is achieved by the interaction of natural base bleed and the reversed flow, which in turn alters the entrainment and development of the shear layer. Kruiswyk and Dutton [21] explored the influence of base cavity on the near-wake field of a two-dimensional body. They found that the structure of vortex street was unaltered; however, vortex street was weakened due to the enhanced mixing at the entrance to the base cavity. This resulted in higher pressures in the near wake for the model with a base cavity. Further, they observed that the shedding frequency of the vortex-street increased marginally relative to the blunt-base configuration. Molezzi and Dutton [22] investigated the near-wake flow field behind a two-dimensional base in a subsonic flow to identify the fluid dynamic mechanisms responsible for the observed base drag reduction in the presence of a base cavity. They argue that the influence of base cavity on the vortex street is related to the expansion and diffusion of the vortices near the cavity, which results in slight reduction in their strength. In the end they conclude that the drag reduction mechanism of the base cavity is due to the physical displacement of the base upstream of the wake region, leading to reduced interaction with the low-pressure vortices. From the above references it is understood that very little attempts have been made with an exception from Dutton and his coworkers to characterize and describe the flow field in the presence of a base cavity. However, in those limited studies that are available, a twodimensional model was considered. As far as the knowledge of present authors is concerned, only single paper is available in the open literature on the base flows of complex geometries such as missile and launch vehicle with a base cavity. Fournier et al. [23] analyzed the cone-cylinder-flare projectile with and without a base cavity at hypersonic speeds numerically. They found that the base cavities are not significantly altering the base drag characteristics; moreover, the larger-length cavities tend to increase the drag.

From the literature cited above it is understood that the base region of a complex model resembling either a true launch vehicle or a missile configurations with the geometric asymmetry in the presence of a base cavity are hardly explored. The purpose of this study is to address this gap. The aim of the work presented in this paper is to understand and characterize the nature of unsteady pressure field in the base region of a typical missile configuration in the presence of a base cavity. The objective is accomplished by carrying out series of experiments in a facility uniquely designed to study the base flow fields. Experiments include unsteady pressure measurements.

\section{Experimental Details}

\section{A. Experimental Facility}

All experiments reported in this paper have been performed in the $0.5 \mathrm{~m}$ axisymmetric base flow tunnel at National Aerospace Laboratories (NAL), India. The blow-down facility was built and has been used over the years specifically to carry experimental studies on the base and afterbody flows without any sting or strut interference [24]. This tunnel has an annular variable throat converging-diverging (c-d) nozzle capable of producing freestream Mach numbers and unit Reynolds numbers in the range of $0.5-3.5$ and $10-50 \times 10^{6} / \mathrm{m}$, respectively. A hollow jet pipe of $127 \mathrm{~mm}$ diameter passes centrally through and is concentric with the c-d nozzle. The jet pipe is cantilevered rigidly far ahead of the nozzle to eliminate interference effects on the downstream flow field. The jet pipe serves couple of purposes; first, it acts as a sting to support the model in the tunnel and, second, it feeds pressurized air into the afterbody nozzles to simulate afterbody jets exhausting from these nozzles. The stagnation pressure of the jet and the freestream can be controlled independently to achieve desired jet and freestream conditions. A fairly well-developed zeropressure gradient turbulent boundary layer is achieved ahead of the base of afterbody [24].

\section{B. Model Configuration}

The model used in the present experimental study is a scale-down afterbody of a typical missile configuration with and without a base cavity. The model without cavity is hereafter referred to as "blunt base" (BB), and the nomenclature of the model mounted with a cavity is provided in Table 1. Figures $1 \mathrm{a}$ and $1 \mathrm{~b}$ show the photographs of the models without and with cavity, respectively, mounted in the base flow facility. It has four control surfaces (fins) oriented in the freestream direction and are fixed rigidly on the circumference of the cylindrical afterbody. The separation angle between the adjacent control surfaces is $90 \mathrm{deg}$. The model also has four wire tunnels, one transportation lock, and two launch shoes to simulate more or less an exact flow field in the presence of these geometric modifications and their effect on base pressure fluctuations. The cylindrical afterbody has a base diameter of $127 \mathrm{~mm}$. The model is mounted at an angle of attack of $0 \mathrm{deg}$, and therefore all the experiments were carried out at this angle of attack. Figure 2 shows the configuration of different cavities tested in the present study. The diameter and thickness of all the cavities are 127 and $1.143 \mathrm{~mm}$, respectively. The ventilated cavities have 12 vent holes equally spaced around the circumference. The diameter of each vent hole is $9.5 \mathrm{~mm}$. The ratio of ventilated area to the base area is 0.067 and is constant for all the cavities. The dimensions of the cavities and the vent holes are chosen based on the guidelines given in [7]. Table 1 provides the nomenclature and dimensions of the cavities tested.

\section{Test Conditions}

Experiments are conducted at a freestream Mach number of $0.7 \pm 0.01$. The desired freestream Mach number is achieved by operating the tunnel at a stagnation pressure of $105.42 \pm 1.3 \mathrm{kPa}$ and the corresponding unit Reynolds number is $11 \times 10^{6} / \mathrm{m}$. The dynamic pressure of the free stream is $26.8 \mathrm{kPa}$.

Table 1 Cavity configurations

\begin{tabular}{lcccc}
\hline \hline S. No. & Type of cavity & Nomenclature & $H, \mathrm{~mm}$ & $H / D$ \\
\hline 1 & Solid & SC & 16.51 & 0.13 \\
2 & Small-length ventilated & SVC & 16.51 & 0.13 \\
3 & Medium-length ventilated & MVC & 33.02 & 0.26 \\
4 & Large-length ventilated & LVC & 54.61 & 0.43 \\
\hline \hline
\end{tabular}




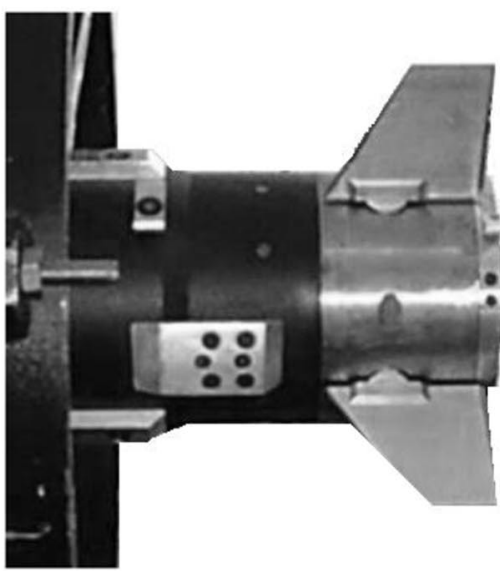

a) Blunt base (BB)

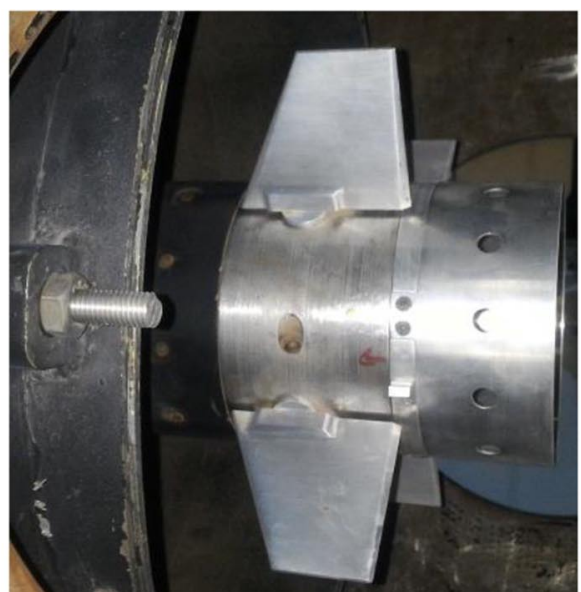

b) Blunt base with cavity

Fig. 1 Photograph of models mounted in the tunnel.
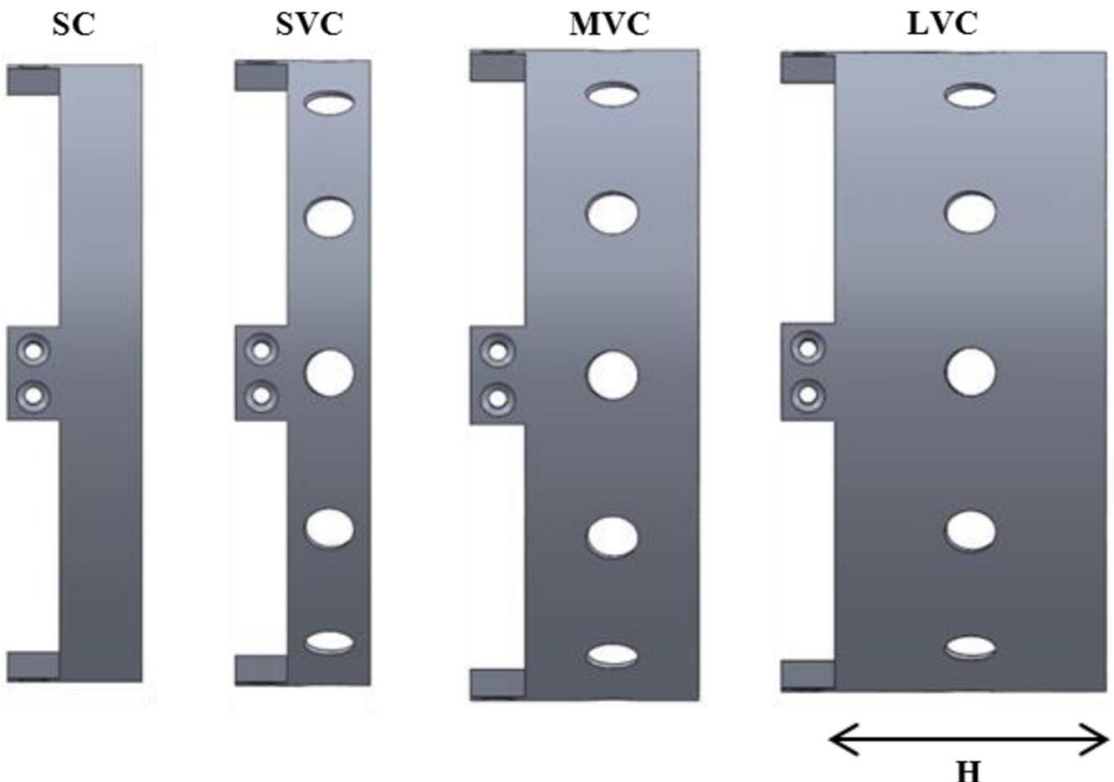

Fig. 2 Configuration of cavities tested in the present study.

\section{Measurements}

Measurement of unsteady pressure is carried out at six circumferential locations on the base of the model. Figure $\underline{3}$ shows the location of unsteady pressure transducers at different azimuthal angles. In addition

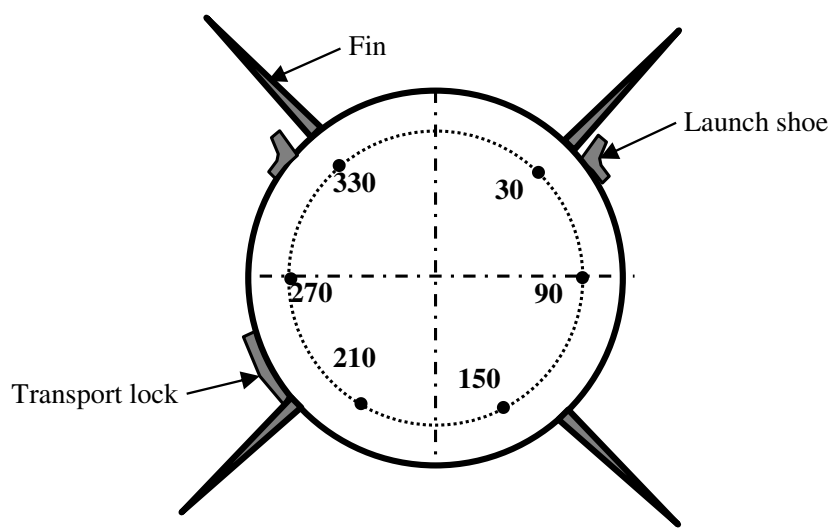

Fig. 3 Schematic of unsteady pressure transducers locations on the base of model. to the unsteady pressure measurement, measurement of stagnation and static pressure was also carried at three locations. Stagnation pressures were measured in the stagnation chamber of the tunnel and the static pressure was measured on the wall of the test section at two different streamwise locations. Stagnation and static pressure data were acquired primarily to monitor whether the desired free stream condition is achieved.

\section{E. Instrumentation}

Kulite pressure transducers (Model XCQ-093-25A and make Kulite Semiconductor Products, Inc., Leonia, NJ) with a full-scale pressure range of $0-172.4 \mathrm{kPa}$ absolute were used to acquire the unsteady pressure signal. These piezoresistive transducers are excited with a 10 V DC supply. The full-scale offset from the transducers is $100 \mathrm{mV} / \mathrm{V}$ with an infinitesimal resolution. The natural frequency of the transducers is $250 \mathrm{kHz}$. The signals from the transducers are initially passed through an operational amplifier with a gain of 10 . The output of the amplifier is thereafter digitized using an NI-PXI-4495 data acquisition card. This card is capable of digitizing 16 analog signals simultaneously with a resolution of 24 bit and having a maximum sampling rate of $205 \mathrm{kS} / \mathrm{s}$. The entire process of data acquisition is controlled by a PC-based NI-Labview software. Static pressures were acquired using a Pressure Systems, Inc., ESP Pressure Scanner Model 
ESP-16TC. The pressure scanner system has 16 silicon piezoresistive pressure transducers fixed on a common substrate and is capable of reading 16 pressures. The pressure scanner is controlled by a PC via an in-house-built interface. The analog signals from the pressure scanner are acquired using National Instruments eight-channel signal conditioner module (SCXI-1520). The analog signals from the signal conditioner are digitized using a 16-bit NI-6036 A/D card. Druckamplified output pressure transducer (Model PMP 4010 and make Druck, Inc., Danbury, CT) with a full-scale pressure range of $1034 \mathrm{kPa}$ absolute was used to acquire and monitor the stagnation pressure of the freestream. This diaphragm transducer is excited with a $24 \mathrm{~V} \mathrm{DC}$ supply and having maximum full-scale output of $10 \mathrm{~V} \mathrm{DC}$. The transducer has an accuracy of $\pm 0.04 \%$ of full scale.

\section{F. Data Acquisition}

Unsteady pressure signals are acquired for a duration of over $8 \mathrm{~s}$ with a sampling rate of $50 \mathrm{kHz}$, resulting in a total of 409,600 data samples from each Kulite in a single run. The complete data from each Kulite were collected in 100 bins with a bin size of 4096 samples. The sampling rate of $50 \mathrm{kHz}$ was chosen based on the Nyquist criterion. The acquired samples are filtered using a digital low-pass filter with a cutoff frequency of $15 \mathrm{kHz}$, because most of the frequencies relevant to the base pressure fluctuations lie below $15 \mathrm{kHz}$.

\section{G. Data Analysis}

Unsteady pressure data acquired were analyzed by computing mean, root mean square (rms), and probability density function (PDF). In addition, autocorrelation, single-point spectrum, and twopoint correlation were also calculated. Power spectral density (PSD) was computed using fast Fourier transform (FFT) algorithm. Before applying FFT, a two-step data-conditioning process has been applied on the data bins to improve the PSD estimates. In the two-step process, the first step involves multiplying the data segments with a Hanning window. This is done to minimize the side-lobe spectral leakage while implementing FFT on the data set. In the second step, the data bins were overlapped by $50 \%$. This has been done to keep the normalized random error to a low value on account of multiplying the data set by a Hanning window in the previous step. The frequency resolution of the PSD obtained with aforementioned window size and the sampling rate is $12.2 \mathrm{~Hz}$. No data-conditioning process has been implemented on the data for computation of autocorrelation and cross-correlation as spectral leakage was not an issue.

\section{H. Uncertainty}

Each measurement is performed at least three times. The experimental uncertainty is calculated based on the repeated independent measurements. It is found that the uncertainty in the pressure measurement is $\pm 2 \%$.

\section{Results}

The results of unsteady pressure data acquired from the Kulite sensors mounted on the base of the model at six circumferential locations for different base cavities are presented and discussed in this section. The spacing between the consecutive measurement locations is $60 \mathrm{deg}$ as shown in Fig. 3 . Though the experiments were conducted at three freestream Mach numbers of $0.5,0.6$, and 0.7 , the results that correspond only to $M_{\infty}=0.7$ were presented. This is because there were no significant conclusions that could be drawn from the results at $M_{\infty}=0.5$ and 0.6 apart from those obtained at $M_{\infty}=0.7$.

Initially, the unsteady pressure data acquired at six locations were time-averaged individually to obtain the mean static pressure. The azimuthal variation in the mean pressure for different base cavity configurations mounted on the base of the missile is plotted and examined. Figure 4 shows the variation in $C p_{b}$ with $\theta$. Significant variation in the mean static pressure is observed with $\theta$. This is because the model used in the present study has a geometric asymmetry ahead of the base region as seen from Fig. 1 and described in the model details section. Deprés et al. [25] reported a mild variation in the static pressure in the azimuthal direction and they

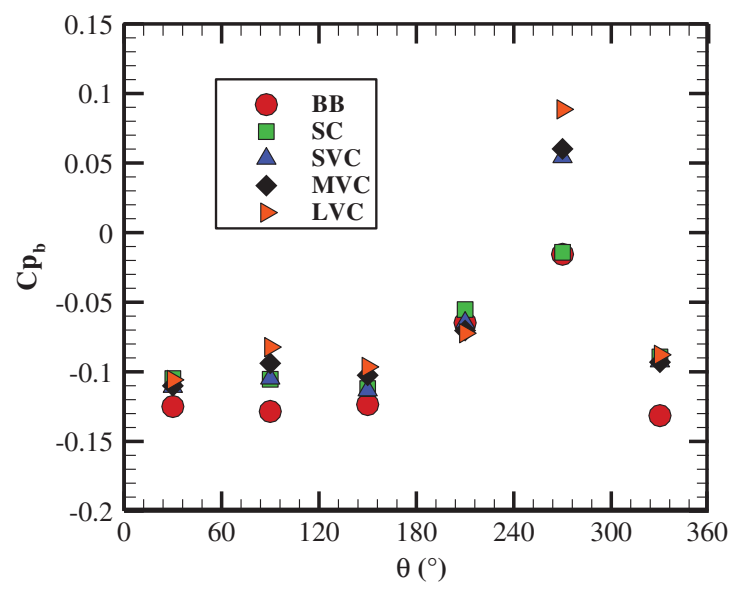

Fig. 4 Azimuthal variation of coefficient of base pressure for different cavity configurations.

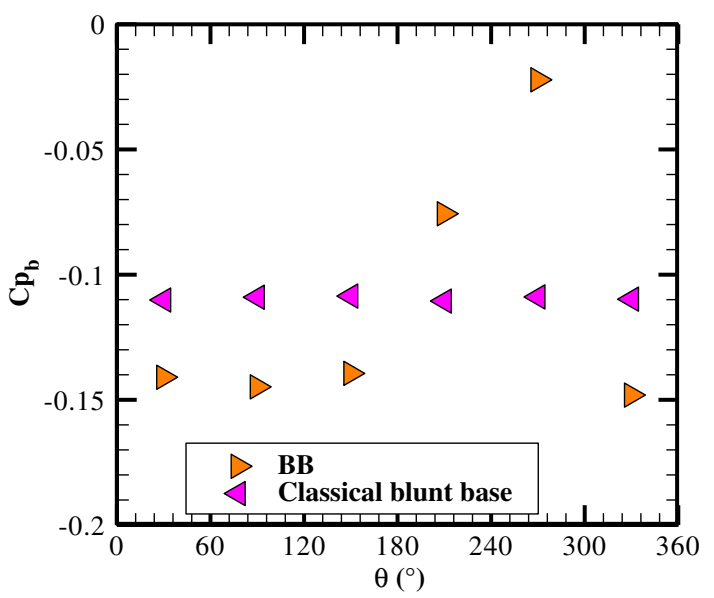

Fig. 5 Azimuthal variation in $C p_{b}$ between $\mathrm{BB}$ and classical BB configuration.

attributed it to the irregularities in the body shape. An axisymmetric model was used in their experimental study at $M_{\infty}=0.85$.

Figure 5 shows the variation in $C p_{b}$ with $\theta$ between $\mathrm{BB}$ model and an axisymmetric BB configuration (i.e., model devoid of any asymmetry) referred to as classical BB in the figure. It is seen that the variation in $C p_{b}$ with $\theta$ for the axisymmetric $\mathrm{BB}$ configuration is negligible. This substantiates that the variation in $C p_{b}$ with $\theta$ for the BB model is due to the additional fittings. The experiment with an axisymmetric BB configuration was carried out in the same facility and under identical blowdown conditions. For the BB model, from Fig. 4 it is seen that the static pressure on the base is below that of the freestream value and significantly higher values of $C p_{b}$ were noticed at azimuthal locations of $\theta=210$ and $270 \mathrm{deg}$. The high value of $C p_{b}$ at these two locations is due to the presence of transportation lock as shown in Fig. 3. The interaction between the separated flow ahead of the transportation lock and the wake flow with the recirculation region at the base could possibly lead to a higher value of base pressure. The average of $C p_{b}$ at all azimuthal locations is -0.112 . This value of $C p_{b}$ is reasonably in agreement with those found in the literature $[1,25,26]$ in view of the asymmetric flow field. The average value of $\overline{C p_{b}}$ at six locations obtained from the current study is compared with that of the Merz et al. [26] as shown in Fig. 6 . It is seen that the results of Merz et al. [26] show a higher value of $C \overline{p_{b}}$ compared with the present study for the corresponding $M_{\infty}$. A blunt axisymmetric model was used in their experiments.

Coming back to Fig. $\underline{4}$, higher values of $C p_{b}$ are noticed for the SC model in comparison with the BB model except at the azimuthal location of $270 \mathrm{deg}$. This is because the base cavity increases the size of 


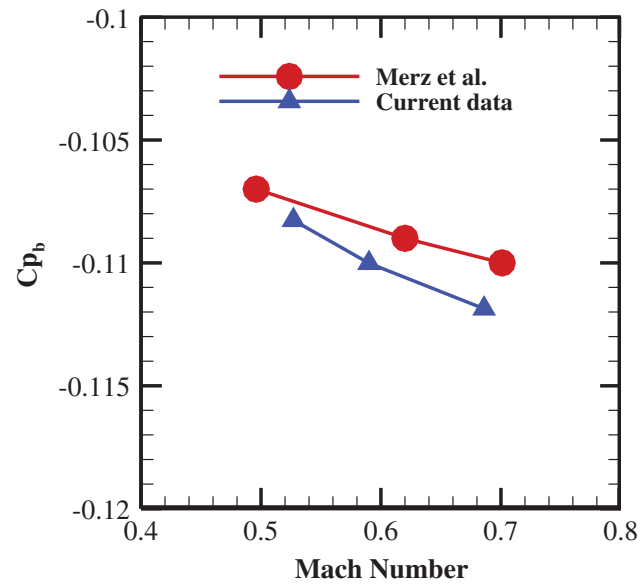

Fig. 6 Comparison of $C p_{b}$ between the present study and that of Merz et al. [26].

recirculation region and also shifts the separation point downstream of the base. This reduces the interaction between the recirculation region and the shear layer. Further, the shifting of the separation point results in less interaction between the base and the low pressure associated with the vortex shedding $[21,22]$. This results in higher values of base pressure. The average of $\overline{C p_{b}}$ at all the azimuthal angles is -0.08 . The ventilated cavities act as a natural bleed devices due to the pressure difference between the freestream flow and the recirculation region [7]. Bleed devices inject the mass of fluid into the base region, thereby increasing the base pressure. For the SVC model, no significant increase in the base pressure is observed compared with the SC model (at corresponding sensor locations) except at the azimuthal location of $270 \mathrm{deg}$. The possible reason for this could be that because the vent holes are normal to the freestream the normal component of the momentum flux of the fluid entering the base region through the vent holes is negligible. This leads to an insignificant amount of mass addition to the recirculation region. In the present study, all the vent holes on the cavities are oriented normal to the freestream flow. So the base pressure increase due to the natural bleed process is negligible. In general, on increasing the length of ventilated cavities from small to large, the base pressure increases due to less interaction between the base and the low pressure associated with the vortex shedding as explained earlier.

The variation in rms of the base pressure fluctuations with $\theta$ is plotted in Fig. 7. It is seen that like $C p_{b}, C p_{\text {rms }}$ also varies with $\theta$. The rms values on the base for the $\mathrm{BB}$ model are higher than that for the model with the base cavities. One possible reason for the reduced rms values could be the reduction in the momentum exchange between the recirculation region and the shear layer in the presence of a base cavity. No appreciable difference in the rms values is observed

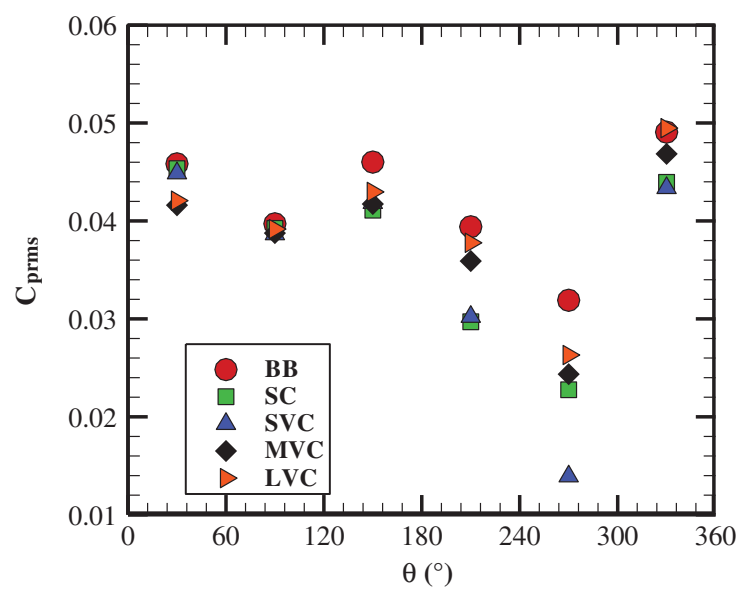

Fig. 7 RMS variation with $\theta$ for different base cavity configurations. between SC and SVC model except at $270 \mathrm{deg}$. On increasing the length of ventilated cavity from small to large, the rms values tend to approach that of the BB model. This could be due to the increase in the pulsing action of the recirculation region as observed from the spectra to be discussed later in this paper.

Figure $\underline{8}$ shows the variation of PDF with $\theta$. All the PDFs have been normalized to ensure that the area under each curve is unity. Each subplot in the figure corresponds to PDFs with different base cavities for a specific value of $\theta$. It is seen from the figure that the peak PDF value corresponding to a specific model varies with $\theta$. This is due to the azimuthal nonuniformity of the base pressure fluctuations on account of flow asymmetry. The lower peak value of PDF is observed for the BB model compared with the model with a cavity. In general, it is noticed that the peak value of PDF is higher for the SC and SVC compared with the MVC and LVC models. Unusual behavior in the PDFs of the model with the base cavities has been observed at azimuthal locations of 210 and $270 \mathrm{deg}$. At these locations the peak value of PDF increases remarkably for the SC and SVC models. In addition to that, a bimodal distribution for the MVC and LVC models is prominently visible at $\theta=270 \mathrm{deg}$. The decrease in the peak value of PDF leads to broadening, and consequently an increase in rms at the base is observed.

The higher-order moments skewness and kurtosis have been examined to further explore the nature of base pressure fluctuations. Figure 9 shows the variation of skewness and kurtosis with $\theta$ for different cavity configurations. It is seen that the skewness varies significantly with $\theta$. The kurtosis, on the other hand, is seen to vary with $\theta$ in the presence of cavity only. In general, it is observed that the skewness decreases in the presence of cavity and reduces further as the cavity length increases. The skewness and kurtosis values for the BB model are higher and very close, respectively, to that of the Gaussian values (Gaussian values: skewness $=0$ and kurtosis $=3$ ). The positive value of skewness at the base for $\mathrm{BB}$ model indicates that the pressure fluctuations above the mean are dominant. In the presence of cavity and upon increasing its length, the skewness decreases and tends toward a Gaussian value. For the MVC and LVC models, skewness at few azimuthal locations on the base goes to a negative value, indicating that the pressure fluctuations below the mean are predominant over those above the mean. This could be due to the increase in the size of the recirculation region on increasing the cavity length, which alters its pulsing behaviour. Kurtosis calculation reveals that it marginally increases for the smaller-length cavities, and thereafter it further decreases (significantly at $\theta=210$ and $270 \mathrm{deg}$ ) with the increase in cavity length for MVC and LVC models. The decrease in kurtosis value indicates that the frequency of occurrence of large-amplitude fluctuations decreases while that of the low-amplitude fluctuations increases. The decrease in value of the kurtosis for MVC and LVC models is due to the increase in the size of the recirculation region, which tries to mitigate the large-amplitude fluctuations.

Spectra of the base pressure fluctuations have been examined to identify the prominent frequency content. Figure 10 shows the spectra at various azimuthal locations for different cavity configurations at the base. The spectra have been normalized with an intent to highlight the important narrow-band frequencies that are associated with the pressure fluctuations.

It is seen that the narrow-band spectra predominantly lie below the Strouhal number of 0.6 at all azimuthal locations. For the BB model, the narrow-band tones in the spectra are observed at Strouhal numbers of $0.0535,0.0802,0.107,0.2,0.408$, and 0.555 . The tones can be classified into three types: 1) type I at Strouhal number of $0.0535,0.0802$, and $0.107 ; 2$ ) type II at 0.2 ; and 3 ) type III at 0.408 and 0.555 . It is to be noted that in type I the tone at 0.107 is a harmonic of that at 0.0535 . Type II tone is associated with the vortex shedding from the shear layer [10]. Further, it is noticed that the three types of tones occur at the same frequencies regardless of their azimuthal location. However, their amplitudes vary depending on the location. For example, the amplitude of type III tones at $\theta=210$ and 270 differs significantly compared with that at the other locations. This is due to the presence of transportation lock that changes the characteristics of the flow that approaches the separation point. For the SC and SVC models the spectra show the absence of type I tones, whereas the type II and III tones are observed to occur at slightly higher 


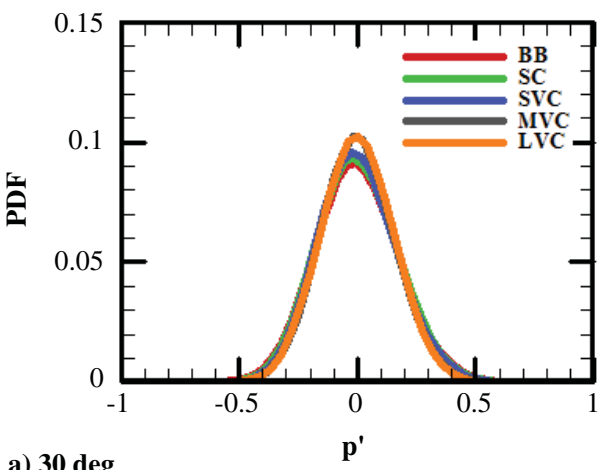

a) $30 \mathrm{deg}$

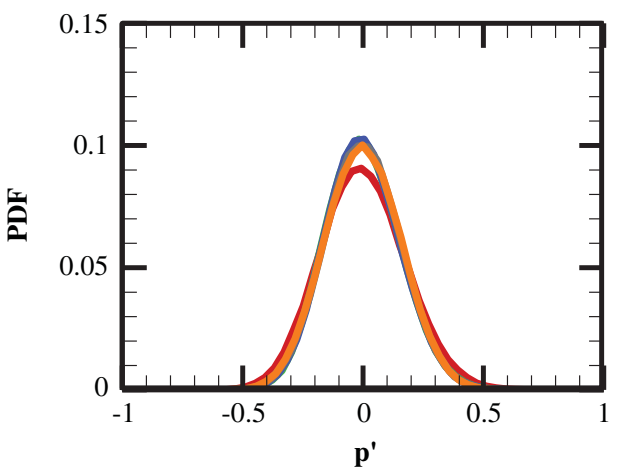

c) $150 \mathrm{deg}$

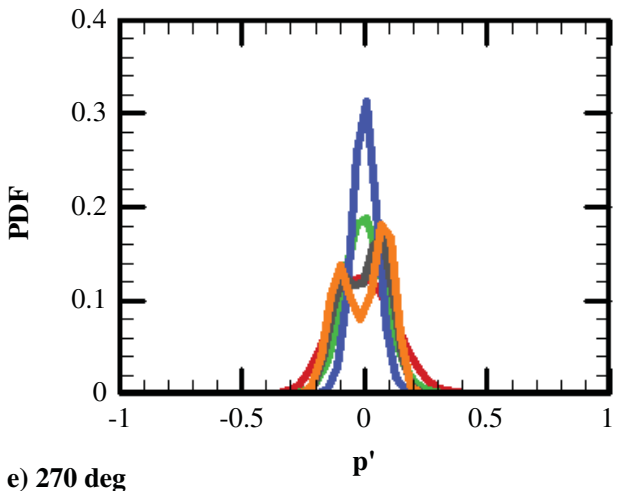

e) $270 \mathrm{deg}$

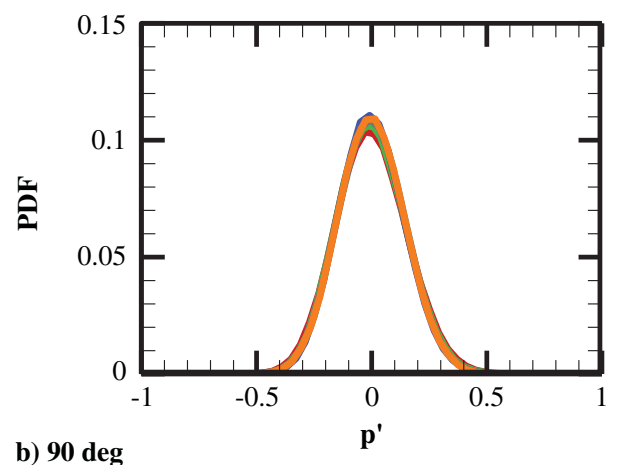

b) $90 \mathrm{deg}$
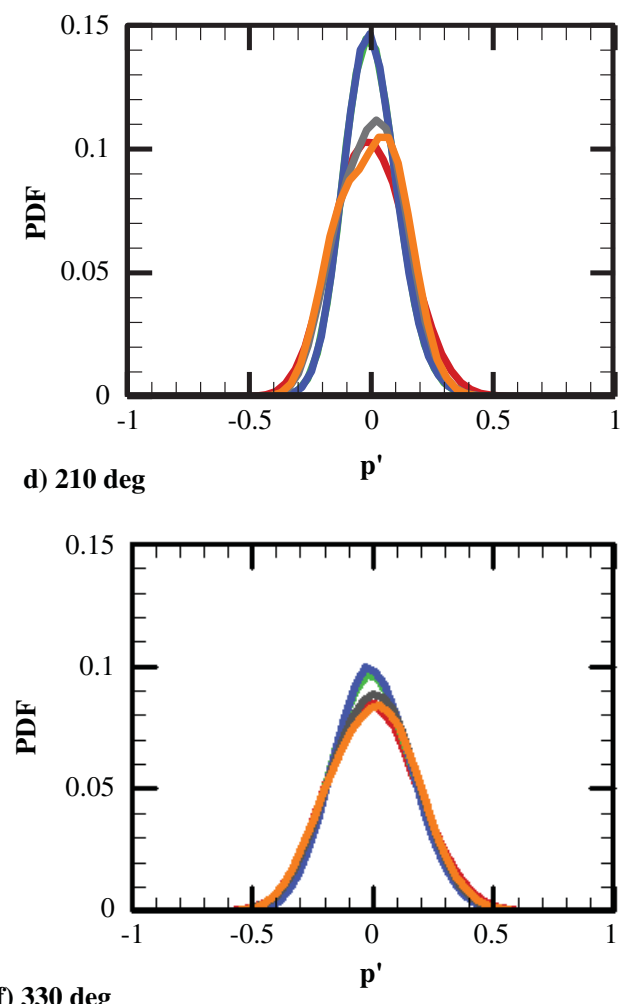

Fig. 8 Variation of PDF with $\theta$ for different base cavity configurations.
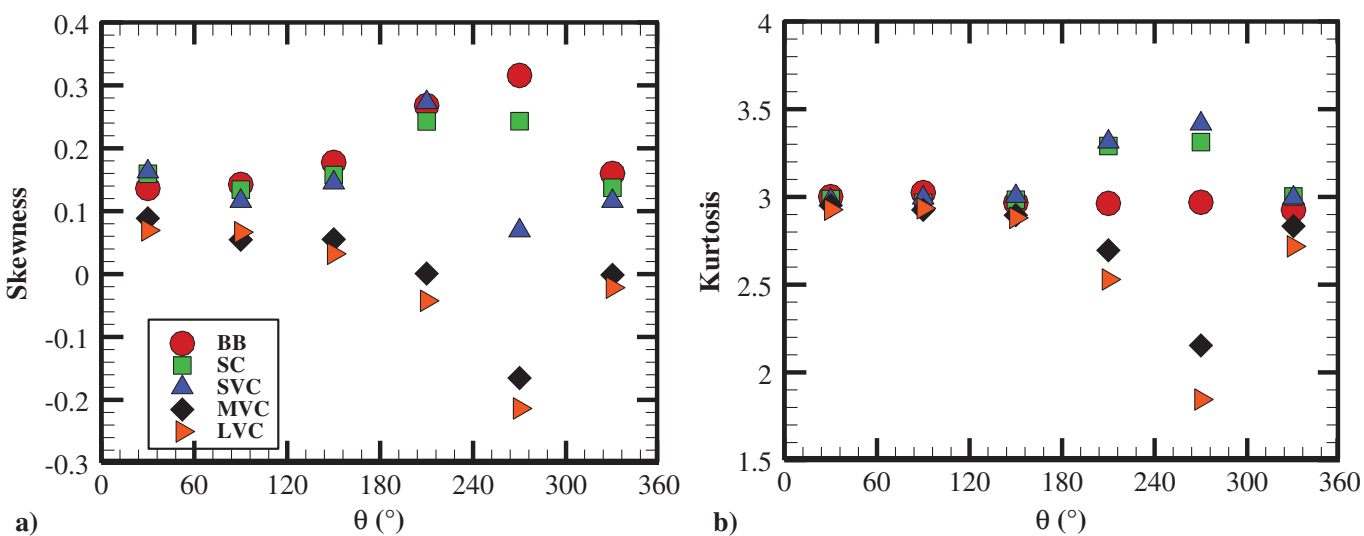

Fig. 9 Variation in skewness and kurtosis with $\theta$ for different cavity configurations.

frequencies. Type II occurs at Strouhal number of 0.207, whereas type III occurs at 0.414 and 0.568 . Similar increase in the frequency of vortex shedding has been observed in earlier studies with base cavities [21,27]. An interesting feature observed in the spectra of these cavities is the absence of all three types of tones at $\theta=270 \mathrm{deg}$. This is due to the transportation lock that alters the flow characteristics as stated earlier. On increasing the length of ventilated cavity to medium and further to large, the type I tones reappear with increased magnitude while the type II tone due to the vortex shedding disappears. The increase in amplitude of the type I tones results in increased rms as observed in Fig. $\underline{7}$. 

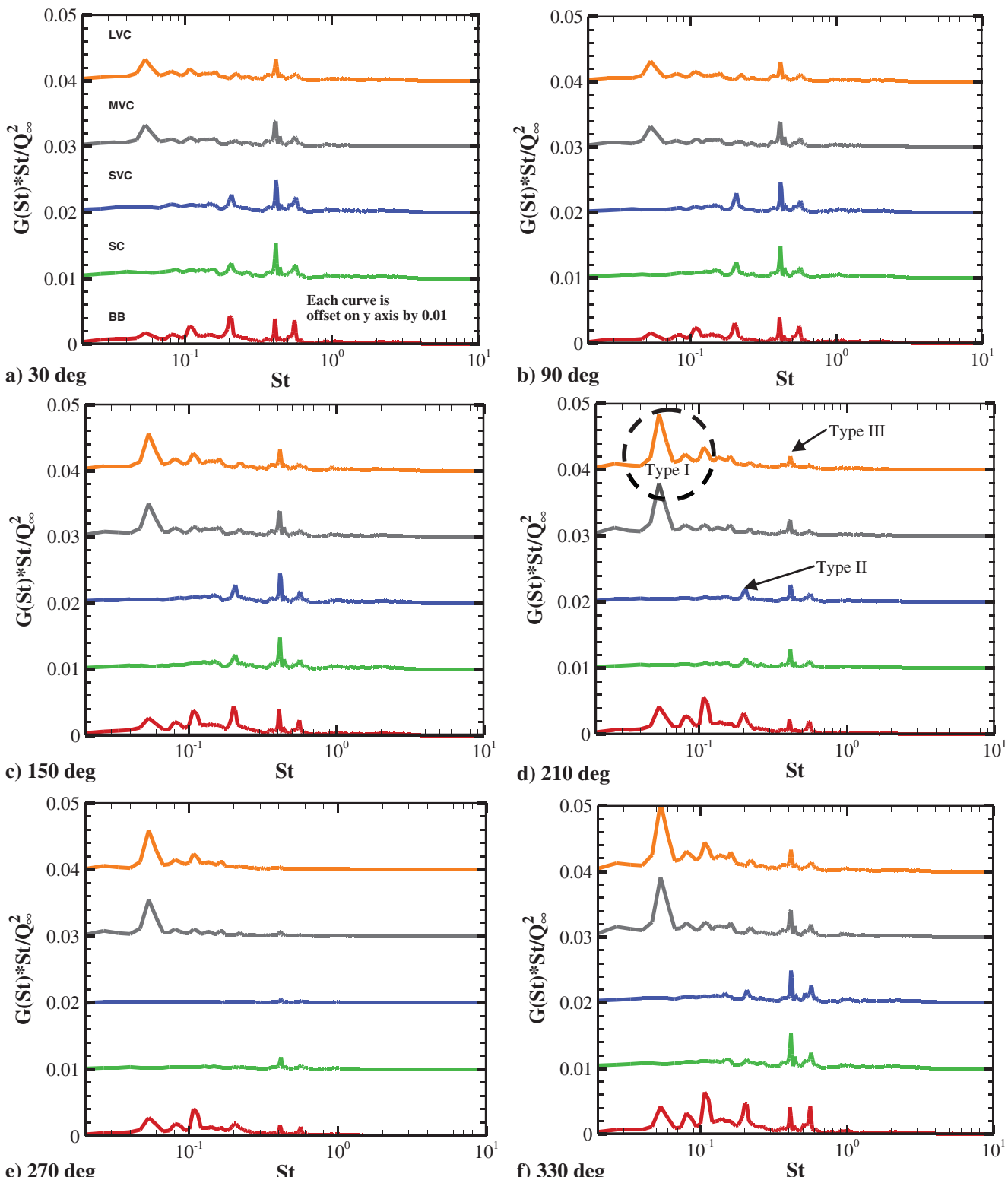

Fig. 10 Spectra with $\theta$ for different cavity configurations.

The disappearance of type II tone could be due to the increased length of the recirculation region that suppresses the disturbance due to the shed vortex from reaching the base. On the other hand, type III tones for MVC and LVC models get suppressed at few locations and get vanished at $\theta=270 \mathrm{deg}$. The shift in frequencies of the type III tones to further higher Strouhal number was not noticed in the models with larger-length cavities.

To gain further insights into the nature of pressure fluctuations, autocorrelation of the pressure signals has been explored. Figure 11 shows the long-time behavior of the autocorrelation at $\theta=30$ and
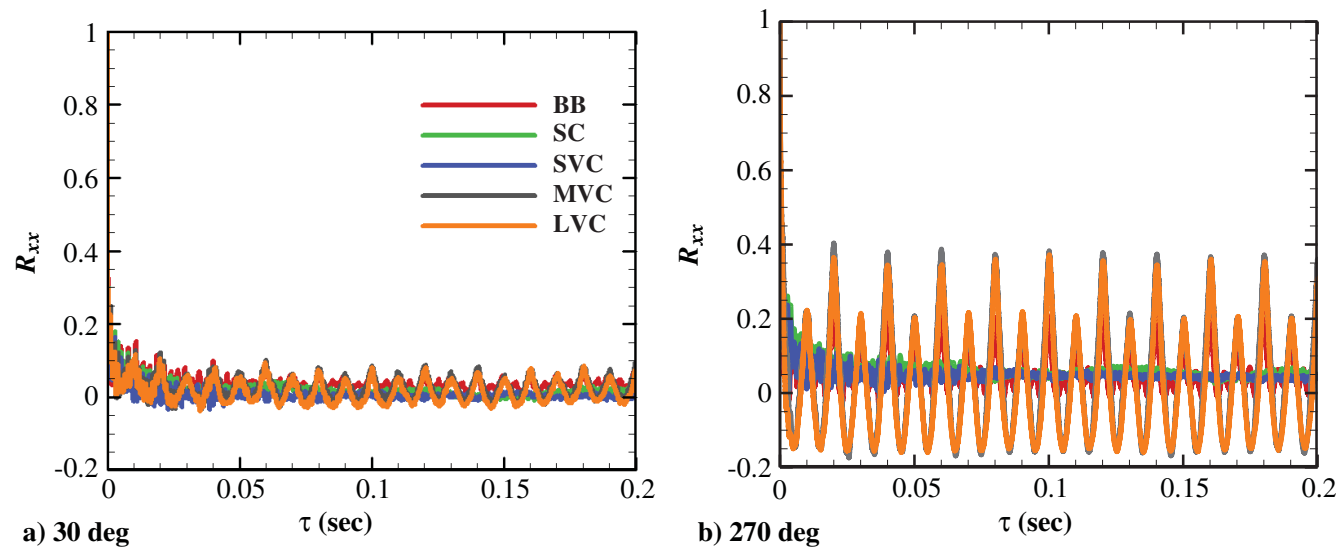

Fig. 11 Plot of autocorrelation coefficient vs time lag for different cavity configurations at $\theta=30$ and $270 \mathrm{deg}$. 

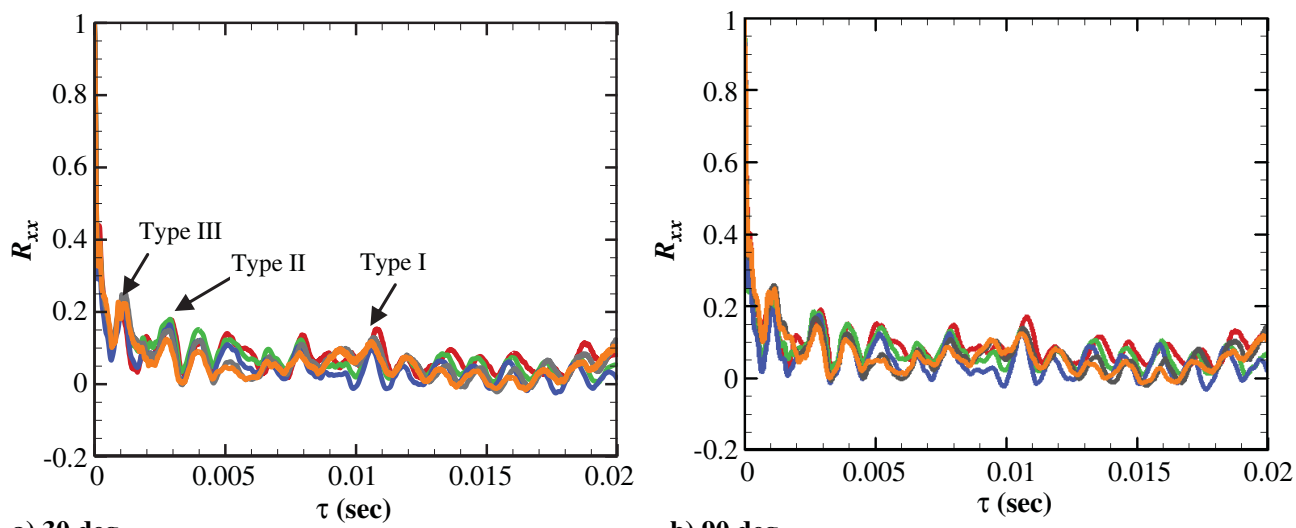

a) $30 \mathrm{deg}$

b) $90 \mathrm{deg}$
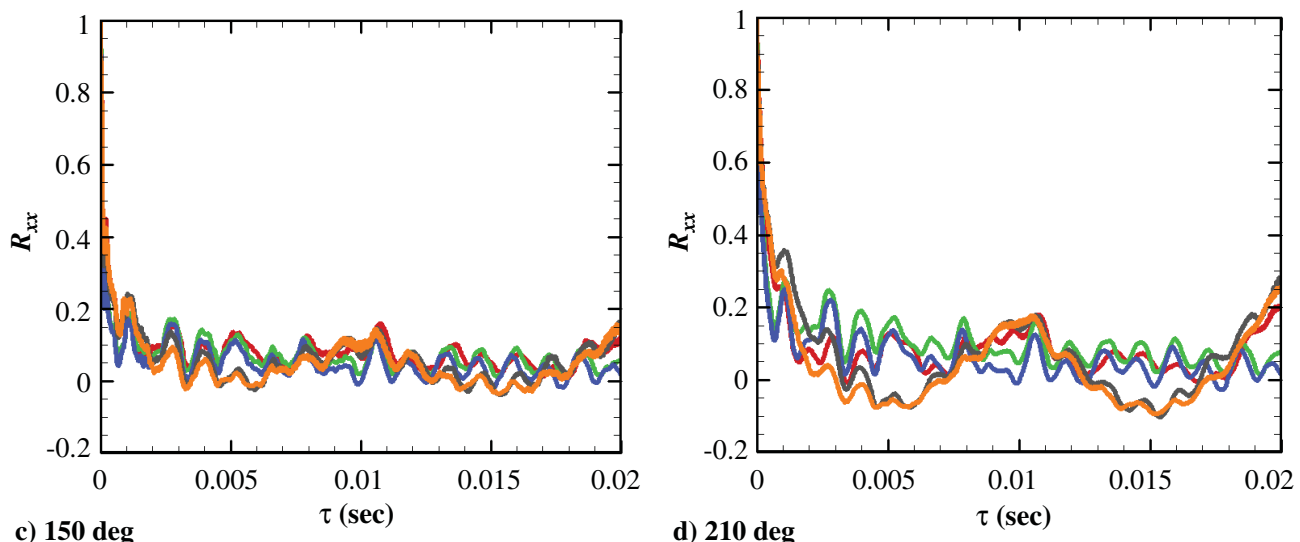

c) $150 \mathrm{deg}$
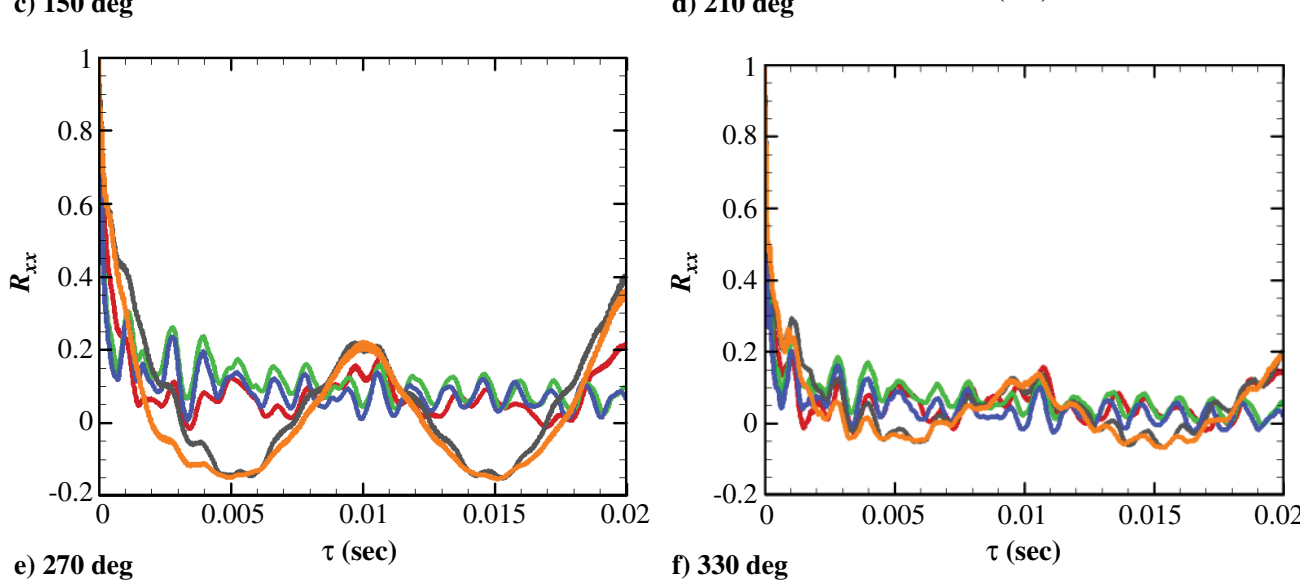

f) $330 \mathrm{deg}$

$\tau(\sec )$

Fig. 12 Plot of autocorrelation coefficient vs time lag for different cavity configurations at various $\theta$.

270 deg. The azimuthal locations are chosen such that they represent the minimum and maximum amplitudes of the oscillations, respectively, in the autocorrelation. The long-time behavior of the autocorrelation shows the presence of periodic oscillations for BB, MVC, and LVC models and virtually no oscillations for SC and SVC models at both the azimuthal locations. This indicates the existence of a highly active mechanism of either recirculation region motion or its pulsing action at the base of BB, MVC, and LVC models.

Such a phenomenon is lacking in SC and SVC models. At $\theta=30 \mathrm{deg}$, the amplitude of the oscillations for $\mathrm{BB}, \mathrm{MVC}$, and LVC models is more or less the same, whereas at $\theta=270 \mathrm{deg}$ the amplitudes are significantly higher for MVC and LVC models compared with the $\mathrm{BB}$ model. A predominant feature of the autocorrelation for the MVC and LVC models is the presence of relatively large negative peaks and is more pronounced at $\theta=270 \mathrm{deg}$. The large negative peaks could be due to the strong pulsing action of the recirculation region. The presence of large negative peaks could be one possible reason for the negative skewness (Fig. 9) observed for these cavities.
Figure 12 shows the short-time behavior of the autocorrelation for various cavity models at all the azimuthal locations. It is seen that all the autocorrelation are wavy. The waviness of the autocorrelation indicates not only the oscillatory behavior of base pressure fluctuations but also the existence of an oscillatory phenomenon responsible for the fluctuations. Further, it is seen that the amplitude of the oscillations decays rapidly and lacks periodicity unlike the long-time behavior. Such a decaying and aperiodic oscillations can be attributed to the local motion or oscillations of the structures close to the base. These unorganized oscillations of the structures are superimposed on the large-scale organized periodic oscillations. The short-time behavior shows no vivid differences among different cavities contrary to the long-time behavior. The three types of tones classified in the spectra are seen as peaks in the autocorrelation as pointed in the figure. An important feature observed in the autocorrelation at $\theta=270 \mathrm{deg}$ is the absence of peaks related to types II and III. This is due to the influence of transportation lock on the flow field before the separation.

Because the unsteady pressure signals are simultaneously acquired from all the six locations, an estimate of cross-correlation between the 

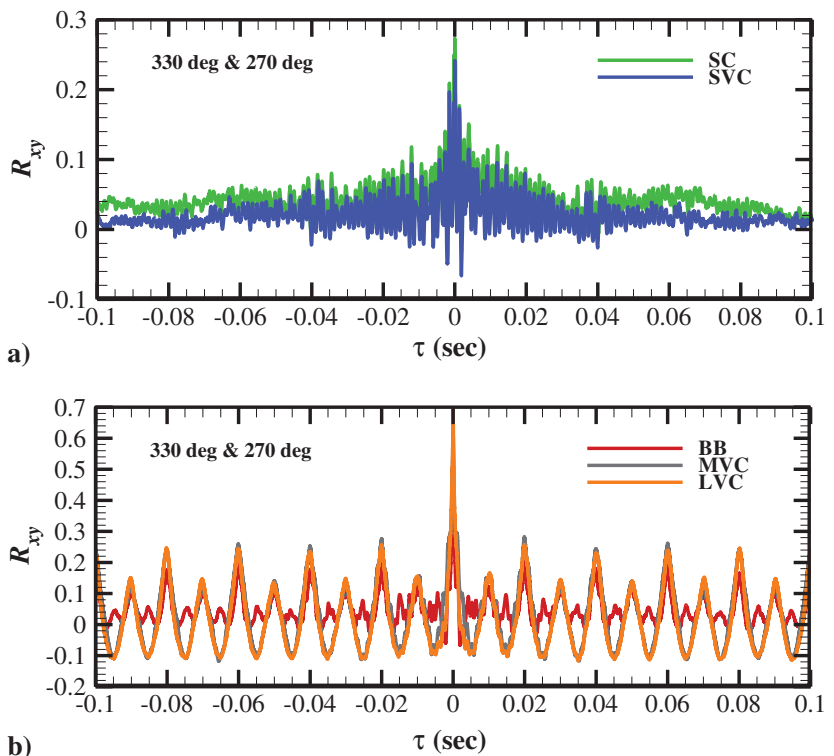

Fig. 13 a) Cross-correlation between $\theta=330$ and $270 \mathrm{deg}$ for $\mathrm{SC}$ and SVC configurations. b) Cross-correlation between $\theta=330$ and 270 deg for BB, MVC, and LVC configurations.

signals is possible. The cross-correlation is obtained between the signals from different azimuthal locations for various cavity models by treating the signal acquired at $\theta=330 \mathrm{deg}$ as the reference. Figure 13 shows the long-time behavior of the cross-correlation between $\theta=3 \overline{30}$ and $270 \mathrm{deg}$ for different cavity models. Figure 13 a shows the long-time behavior of the cross-correlation for SC and SV $\overline{\mathrm{C}}$ models. It is seen that for both the models aperiodic oscillations of moderate amplitude persist in the cross-correlation for a short duration. As the separation time increases the amplitude of the oscillations diminishes rapidly, and above $0.04 \mathrm{~s}$ the cross-correlation displays a behavior characteristic of a random noise signal. Figure $13 \mathrm{~b}$ shows the long-time behavior of the cross-correlation for BB, MVC, and LVC models. The cross-correlation of all the three models exhibits a large-amplitude periodic oscillation that exists the entire time duration for which the signal is acquired. This is in sharp contrast to that observed in the aforementioned cavity models. Further, it is seen that the oscillations are periodic. The oscillations above the time lag of $0.02 \mathrm{~s}$ are more or less a repetition of those observed at and below $0.02 \mathrm{~s}$. For the MVC and LVC models, a large negative peak is noticed in the cross-correlation similar to what has been observed in the autocorrelation probably for the same reason. A similar long-time behavior is observed for the other combinations of $\theta$.

Figure 14 shows a sequence of cross-correlation plots between $\theta=330 \overline{\operatorname{deg}}$ and $\theta=30,90,150,210$, and $270 \mathrm{deg}$, respectively, for different cavity models. The maximum value of the cross-correlation is observed for the LVC model for all combinations of $\theta$ and that the maximum value reduces with the decrease in length of the cavity for the models with ventilated cavities. Further, the maximum value for a specific cavity model varies with the azimuthal spacing between the correlated locations. It is seen for the BB, MVC, and LVC models that the maximum cross-correlation occurs at zero time lag for all the combinations of $\theta$. Because the maximum cross-correlation is observed at zero time lag, it can be suggested that this could be attributed to the global mechanism of recirculation region motion/ pulsing in concurrence with the shear-layer flapping. At higher time lags the magnitude of the cross-correlation undergoes low-frequency oscillations of very high amplitude. A typical picture of the long-time behavior of the oscillations is shown in Fig. 13b and has been discussed. Unlike the previously discussed models, the maximum cross-correlation for the SC and SVC models is not observed at zero time lag for all the combinations of $\theta$. This indicates that the global mechanism of recirculation region motion/pulsing is absent in these cavity models. At higher time lags the magnitude of cross-correlation exhibits a behavior typical to that observed in uncorrelated signals as shown in Fig. 13a. The low-frequency oscillations observed for the
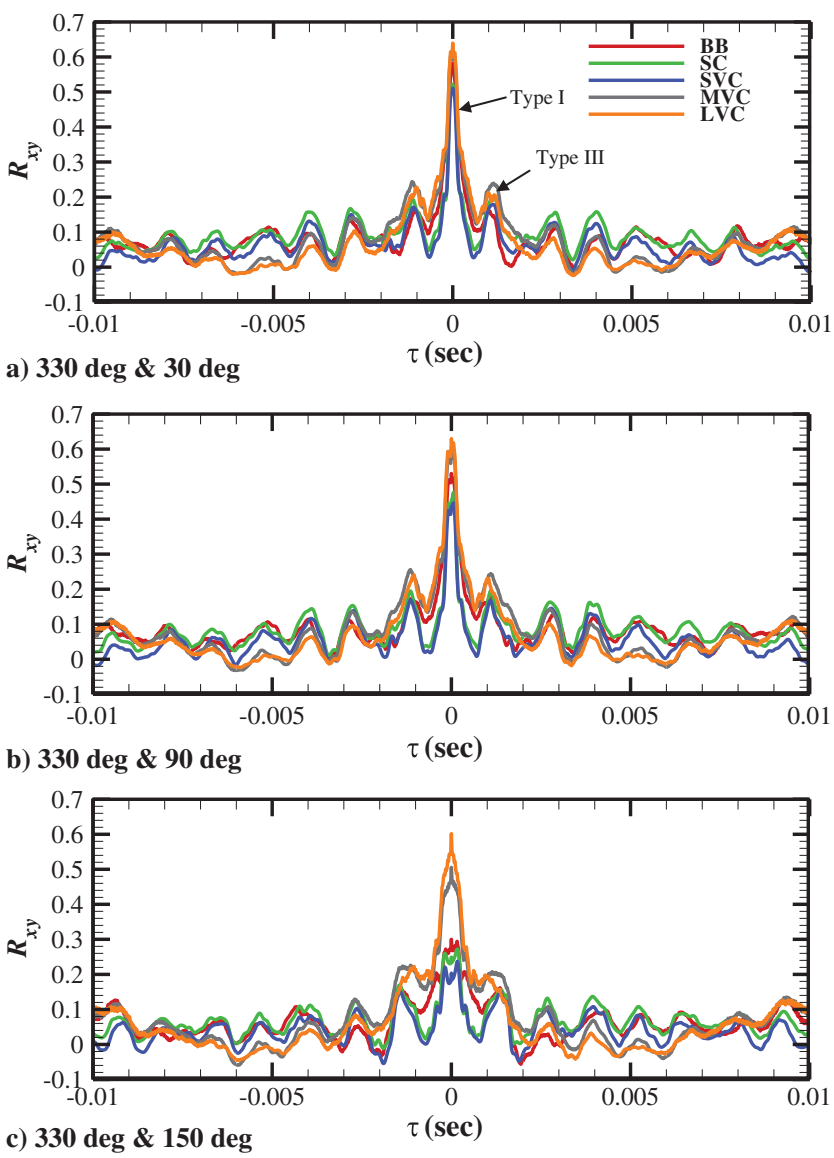

deg \& 150 deg

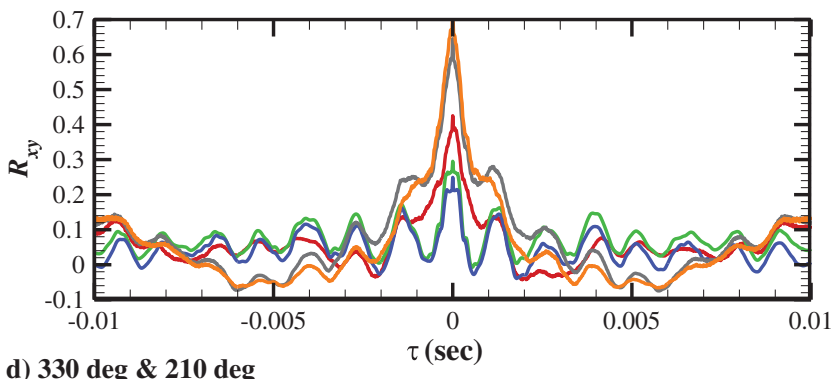

d) $330 \operatorname{deg} \& 210 \mathrm{deg}$

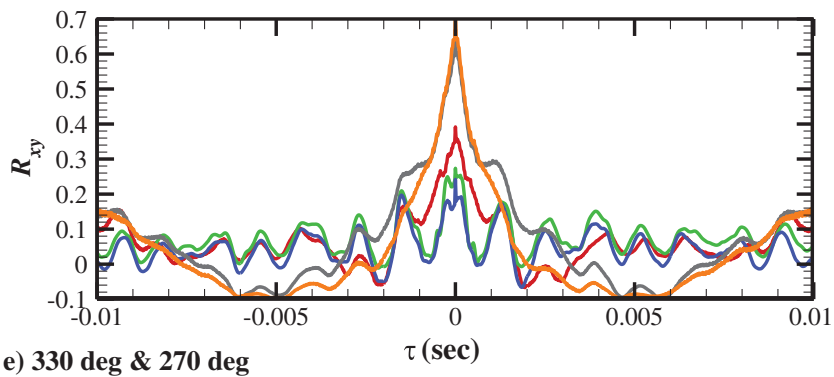

Fig. 14 Cross-correlation between $\theta=330 \mathrm{deg}$ and other azimuthal locations for different cavity configurations.

BB, MVC, and LVC models are unnoticed in the SC and SVC models. The Strouhal number corresponding to the low-frequency oscillation and its harmonics associated with the global mechanism occur below the Strouhal number of 0.12 for the BB, MVC, and LVC models. If one looks at the spectra of these models it is seen that the type I tones occur below the Strouhal number of 0.12 at all the azimuthal locations. This indicates that the type I tones are due to the global mechanism of recirculation region pulsing/motion. For the SC and SVC models, because the global mechanism is absent, the spectra of these models do not display type I tones. Similar to the autocorrelation, the magnitude of the cross-correlation for BB, MVC, and LVC models decreases from its peak value and exhibits aperiodic small-amplitude oscillations 
before undergoing the low-frequency oscillations of very high amplitude at higher time lags as discussed above. The SC and SVC models are also seen to display an aperiodic oscillation.

In addition to the above-discussed oscillation associated with the recirculation region, a secondary hump superimposed on the primary cross-correlation curves is noticed between the time lags $9.8 \times 10^{-4}$ and $15 \times 10^{-4} \mathrm{~s}$ depending on the combinations of $\theta$ chosen and on the type of base cavity. The associated Strouhal numbers range from 0.365 to 0.56 . Because the secondary hump in the correlation curves is observed at a finite time lag (not zero time lag) it indicates the presence of motions, possibly of large turbulent structures or vortex very close to the base within the recirculation region. Further, because the secondary hump is noticed for all combinations of $\theta$, it can be said that the motion of the structures does occur in all directions. It is seen earlier that the spectra in Fig. 10 exhibit two high-amplitude tones (type III) at Strouhal numbers $\overline{0.4}$ and 0.55 , and they lie within the above-said range. Moreover, it can be said that the high-amplitude tones observed at Strouhal numbers 0.4 and 0.55 in the spectra could be because of these locomotions. Deck and Thorigny [28] and Kawai and Fujii [1] have shown the presence of large-scale structures and secondary vortex close to the base in their respective computational studies. A well-defined secondary hump discussed above is not seen for the LVC model in the cross-correlation plot between $\theta=330$ and $210 \mathrm{deg}$ and between $\theta=330$ and $270 \mathrm{deg}$.

\section{Discussion}

The significant results obtained from the above analysis of unsteady pressure are presented in Table 2 . Table 2 shows the various quantities of interest averaged over all the six transducer locations. In the table, the $C p_{b}$ and $C p_{\text {rms }}$ of various cavity configurations have been compared with the BB model, whereas the skewness and kurtosis are compared with that of the Gaussian value (i.e., difference with the Gaussian value).

It is seen from the table that as the length of the base cavity increases the net value of the base pressure $C p_{b}$ increases. On the other hand, the reduction in the overall unsteadiness decreases with the increase in the length of the base cavity as observed from the $C p_{\text {rms }}$ values. However, the frequency of large-amplitude fluctuations decreases with the increase in the length of the cavity as seen from the kurtosis. On relating the physical quantities calculated with different types of tones and in turn the mechanisms driving them, it is seen that the enhancement of base pressure and reduction in the frequency of large-amplitude fluctuations for the MVC and LVC configurations is probably due to the suppression of vortex shedding mechanism at the base. This further suggests that the dominant mechanism responsible for the base drag is the vortex shedding. In the case of SC and SVC, the reduction in the overall unsteadiness at the base is possibly due to the absence of pulsing action of the recirculation region. This indicates that the pulsing action of the recirculation region is responsible for the overall unsteadiness at the base. Based on the preceding discussion the optimum cavity length for a specific application depends on the motive. If the motive is to reduce the base drag, cavities with a large $H$ value should be selected over the small $H$ cavities. However, if the motive is to reduce the unsteadiness and the unsteady loads, then one should prefer cavities with a small $H$ value over the larger value. Further, one should bear in mind the weight aspect while selecting cavities with a large $H$ for base drag reduction. Coming to the question of choice between a solid cavity and a ventilated cavity, cavity with the ventilation should be preferred due to its inherent advantages as presented in Table 2. However, for the ventilated cavities having the same ratio of ventilated area to the base area but differ in the number of vent holes and area of the holes, the behavior of the cavities and their influence on the base flow field could vary due to the difference in the rate of momentum transfer through the vent holes. A further study is needed to ascertain the impact of a number of vent holes and their size on the flow field at the base.

\section{Conclusions}

An experimental study was carried out to investigate the effect of the base cavity on the pressure fluctuations in the base region of a typical missile configuration. The aim of the study was to characterize the pressure fluctuations and to identify the dominant mechanisms driving them. Experiments were conducted at a freestream Mach number of 0.7. Unsteady pressure measurements at six azimuthal locations were performed as a part of the study.

The characteristics of base pressure fluctuations vary significantly along the azimuthal direction due to the nonuniformity of flow field on account of asymmetry of the model. The base pressure is enhanced in the presence of base cavity. This is due to the reduction in the interaction between the recirculation region and the shear layer and also due to the mitigated effect of low pressure associated with the vortex shedding on the base plane. The base pressure increases with the increase in base cavity length. The base pressure remains more or less unaltered due to the ventilation because the vent holes are normal to the freestream, leading to less entrainment of fluid from the freestream into the base region (Fig. 4). However, the average value of the pressure on the base is seen to improve in the presence of a ventilated cavity (Table 2). The rms of the pressure fluctuations is observed to decrease in the presence of base cavity. This is because of the reduction in momentum exchange between the recirculation region and the shear layer. However, it gets enhanced as the length of the base cavity increases. This is due to stronger pulsing action of the recirculation region. The probability density function (PDF) of the pressure fluctuations indicate an increase in peak value of PDF in the presence of a cavity. The skewness decreases in the presence of base cavity and further reduces as the length of the cavity is increased. Kurtosis is seen to reduce as the length of the cavity increases. This is due to the increase in size of the recirculation region, which alters its characteristics and mitigates the frequency of occurrence of large-amplitude oscillations.

Spectra of pressure fluctuations on the "blunt base" (BB) model exhibit three types of tones based on Strouhal number. For LVC and MVC models the spectra show type I and III tones while the type II tone is suppressed. On the other hand, for SC and SVC models spectra show type II and III while the type I tone is suppressed. The long-time behavior of the auto- and cross-correlation plots exhibits periodic largeamplitude, low-frequency oscillations for the BB, MVC, and LVC models, whereas such oscillations are not observed for SC and SVC models. Further, the cross-correlation indicates that the low-frequency oscillations are due to the strong pulsing action of the recirculation region. This suggests that beyond a certain length of the base cavity the dominant mechanism is the pulsing action of the recirculation region. Further, the type I tone observed in the spectra is due to the pulsing action of the recirculation region. The short-time behavior of both auto- and cross-correlation of all the cavities exhibits aperiodic oscillations. Cross-correlation indicates that this aperiodic oscillation is due to the oscillation/motion of large structures or vortices within the recirculation region. It can be said that the type III tone observed in the spectra is due to the motion of large structures within the recirculation region. Overall it can be concluded that the mechanisms that drive the base pressure fluctuations are the pulsing action of the recirculation region and large structure convection within the recirculation region for large cavities,

Table 2 Summary of significant results

\begin{tabular}{lccccccc}
\hline \hline Configuration & $C p_{b}, \%$ & $C p_{\text {rms }}, \%$ & Skewness & Kurtosis & Type I & Type II & Type III \\
\hline BB & 0 & 0 & 0.2 & -0.024 & $\checkmark$ & $\checkmark$ & $\checkmark$ \\
SC & 18.3 & -11.8 & 0.18 & 0.091 & $\times$ & $\checkmark$ & $\checkmark$ \\
SVC & 26.6 & -15.5 & 0.147 & 0.118 & $\times$ & $\checkmark$ & $\checkmark$ \\
MVC & 30.4 & -9 & 0.0054 & -0.257 & $\checkmark$ & $\times$ & $\checkmark$ \\
LVC & 39.5 & -5.6 & -0.018 & -0.361 & $\checkmark$ & $\times$ & $\checkmark$ \\
\hline \hline
\end{tabular}


whereas for the small cavities it is the influence of vortex shedding and large structure convection. From the authors' view point it is suggested that small-length ventilated cavities can be preferred over the largelength ventilated cavities for mitigating the overall unsteadiness at the base. Further, it can be stated that the dominant mechanism driving the overall unsteadiness at the base is the pulsing action of the recirculation region. However, the large-amplitude fluctuations and the base drag can be reduced by suppressing the vortex shedding using a large-length ventilated cavity over the small ones.

\section{Acknowledgments}

The authors would like to express their sincere gratitude to A. Narayanaswami, V. Biju, A. Narayana, G. Ramachandra, and S. M. Mohan Kumar for their unstinted support during model fabrication and experimental campaign. The authors would also like to express their sincere gratitude to DRDL for financially supporting this project. The authors wish to express their deep sense of gratitute to the anonymous reviewer for offering valuable suggestions to improve the quality of the paper.

\section{References}

[1] Kawai, S., and Fujii, K., "Time-Series and Time-Averaged Characteristics of Subsonic to Supersonic Base Flows," AIAA Journal, Vol. 45, No. 1, 2007, pp. 289-301. doi: $10.2514 / 1.24601$

[2] Janssen, J. R., and Dutton, J. C., "Time-Series Analysis of Supersonic Base-Pressure Fluctuations," AIAA Journal, Vol. 42, No. 3, 2004, pp. 605-613. doi: $10.2514 / 1.4071$

[3] Simon, F., Deck, S., Guillen, P., Cayzac, R., and Merlen, A., "ZonalDetached-Eddy Simulation of Projectiles in the Subsonic and Transonic Regimes," AIAA Journal, Vol. 45, No. 7, 2007, pp. 1606-1619. doi:10.2514/1.26827

[4] Eldred, K. M., "Base Pressure Fluctuations," The Journal of the Acoustical Society of America, Vol. 33, No. 1, 1961, pp. 59-63. doi:10.1121/1.1908404

[5] Panov, Y. A., Shvets, A. I., and Khazen, A. M., "Study of Base Pressure Fluctuations Behind a Cone in Supersonic Flow," Fluid Dynamics, Vol. 1, No. 6, 1966, pp. 84-86. doi:10.1007/BF01022287

[6] Saile, D., Gülhan, A., Henckels, A., Glatzer, C., Statnikov, V., and Meinke, M., "Investigations on the Turbulent Wake of a Generic Space Launcher Geometry in the Hypersonic Flow Regime," EUCASS Progress in Flight Physics, Vol. 5, EDP Sciences, June 2013, pp. 209-234. doi:10.1051/eucass/201305209

[7] Viswanath, P. R., "Flow Management Techniques for Base and Afterbody Drag Reduction," Progress in Aerospace Sciences, Vol. 32, Nos. 2-3, 1996, pp. 79-129. doi:10.1016/0376-0421(95)00003-8

[8] Cannon, P. M., Elliott, G. S., and Dutton, J. C., "Time-Series Axisymmetric Base Pressure Measurements with Simultaneous NearWake Planar Visualizations," AIAA Paper 2005-5285, 2005.

[9] Weiss, P. É., Deck, S., Robinet, J. C., and Sagaut, P., "On the Dynamics of Axisymmetric Turbulent Separating/Reattaching Flows," Physics of Fluids, Vol. 21, No. 7, 2009, Paper 075103. doi:10.1063/1.3177352

[10] Schrijer, F. F. J., Sciacchitano, A., and Scarano, F., "Spatio-Temporal and Modal Analysis of Unsteady Fluctuations in a High-Subsonic Base Flow," Physics of Fluids, Vol. 26, No. 8, 2014, Paper 086101. doi:10.1063/1.4891257

[11] Choi, H., Jeon, W. P., and Kim, J., "Control of Flow over a Bluff Body," Annual Review of Fluid Mechanics, Vol. 40, No. 1, 2008, pp. 113-139. doi:10.1146/annurev.fluid.39.050905.110149
[12] Zdravkovich, M. M., "Review and Classification of Various Aerodynamic and Hydrodynamic Means for Suppressing Vortex Shedding," Journal of Wind Engineering and Industrial Aerodynamics, Vol. 7, No. 2, 1981, pp. 145-189. doi:10.1016/0167-6105(81)90036-2

[13] Nash, J. F., A Discussion of Two-Dimensional Turbulent Base Flows, ARC, London, R\&M No. 3344, 1967, pp. 1-39.

[14] Viswanath, P. R., and Patil, S. R., "Effectiveness of Passive Devices for Axisymmetric Base Drag Reduction at Mach 2," Journal of Spacecraft and Rockets, Vol. 27, No. 3, 1990, pp. $234-237$. doi: $10.2514 / 3.26130$

[15] Jackson, R., Wang, Z., and Gursul, I., "Afterbody Drag Reduction Using Active Flow Control," 55th AIAA Aerospace Sciences Meeting, AIAA Paper 2017-0954, 2017.

[16] Weiss, P. É., and Deck, S., "Control of the Antisymmetric Mode $(m=1)$ for High Reynolds Axisymmetric Turbulent Separating/ Reattaching Flows," Physics of Fluids, Vol. 23, No. 9, 2011, Paper 095102 . doi:10.1063/1.3614481

[17] Bearman, P. W., "The Effect of Base Bleed on the Flow Behind a TwoDimensional Model with a Blunt Trailing Edge," Aeronautical Quarterly, Vol. 18, No. 3, 1967, pp. 207-224. doi:10.1017/S0001925900004212

[18] Van Leeuwen, P. M., "Computational Analysis of Base Drag Reduction Using Active Flow Control," Master of Science Thesis, Delft Univ. of Technology, Delft, The Netherlands, 2009.

[19] Morel, T., "Effect of Base Slant on Flow in the Near Wake of an Axisymmetric Cylinder," Aeronautical Quarterly, Vol. 31, No. 2, 1980, pp. 132-147. doi:10.1017/S0001925900010957

[20] Compton, W. B., III, "Effect on Base Drag of Recessing the Bases of Conical Afterbodies at Subsonic and Transonic Speeds," NASA TN D-4821, 1968.

[21] Kruiswyk, R. W., and Dutton, J. C., "Effects of a Base Cavity on Subsonic Near-Wake Flow," AIAA Journal, Vol. 28, No. 11, 1990, pp. $1885-1893$. doi: $10.2514 / 3.10495$

[22] Molezzi, M. J., and Dutton, J. C., "Application of Particle Image Velocimetry in High-Speed Separated Flows," AIAA Journal, Vol. 31, No. 3, 1993, pp. 438-446.

doi:10.2514/3.11349

[23] Fournier, E. Y., Dupuis, A. D., and Edwards, J. A., "Base Cavity Effects on the Aerodynamic Characteristics of a Hypersonic Flared Projectile," Journal of Spacecraft and Rockets, Vol. 34, No. 6, 1997, pp. 737-743. doi: $10.2514 / 2.3304$

[24] Mathur, N. B., and Viswanath, P. R., "Drag Reduction from Square Base Afterbodies at High Speeds," Journal of Aircraft, Vol. 41, No. 4, 2004, pp. 811-820. doi:10.2514/1.532

[25] Deprés, D., Reijasse, P., and Dussauge, J. P., "Analysis of Unsteadiness in Afterbody Transonic Flows," AIAA Journal, Vol. 42, No. 12, 2004, pp. 2541-2550. doi: $10.2514 / 1.7000$

[26] Merz, R. A., Page, R. H., and Przirernbel, C. E. G., "Subsonic Axisymmetric Near-Wake Studies," AIAA Journal, Vol. 16, No. 7, 1978, pp. 656-662. doi: $10.2514 / 3.60954$

[27] Clements, R. R., "Computer Models of Separated Flows Behind Two Dimensional Bluff Bodies," Doctoral Dissertation, Univ. of Cambridge, Cambridge, England, U.K., 1973.

[28] Deck, S., and Thorigny, P., "Unsteadiness of an Axisymmetric Separating-Reattaching Flow: Numerical Investigation," Physics of Fluids, Vol. 19, No. 6, 2007, Paper 065103. doi: $10.1063 / 1.2734996$

M. S. Miller Associate Editor 